\title{
Modeling and Simulation in Atom Probe Tomography
}

\author{
D. J. Larson ${ }^{1}$ and M. P. Moody ${ }^{2}$ \\ ${ }^{1}$ Cameca Instrument Inc., 5470 Nobel Drive, Madison, WI 53711, USA \\ 2 Department of Materials, University of Oxford, Parks Road, Oxford, OXI 3PH, UK
}

Atom probe tomography (APT) is a characterization technique based on electrostatic field evaporation, mass spectrometry and point projection microscopy. APT generates an atom-by-atom image of the specimen, in which each atom is chemically identified and precisely located in three dimensions [1]. As such, the form of the APT data, is highly complementary to a wide range of modeling and simulation approaches.

The use of computers to facilitate modeling, simulation, and data collection / processing has been widespread over the history of the development and implementation of the atom probe. Modeling has provided new insights and underpinned advances in all aspects of the technique; from our understanding of the physics of the field evaporation process, to probing the origins of limitations in the state-of-the-art image reconstruction algorithms, and ultimately the interpretation of the acquired data. Some examples include: Field Ion Microscopy (FIM) image simulation [2,3], computerized experiment control [4], voltage pulsing [5] and laser heating [6-10], first principles field ionization and evaporation calculations [11,12], data analysis [13-15], ion trajectory simulations [16], just to name a few. (Note that this list is by no means exhaustive, but is simply intended to highlight the broad range of topics addressed by these techniques.)

This tradition of combining modeling and simulation with experiment remains at the forefront of advancing the APT technique. In this volume we have invited several distinguished scientists to present their recent research across a range of topics, all of them tied together through the development of 
modeling techniques to provide critical new insights on some aspect of atom probe tomography.

In the first article in this volume, M. Karakha and H. Kreuzer discuss the kinetics of field evaporation, as applied to semiconductors, using density functional theory and obtain good agreement with experimental results for silicon. The second contribution is from F. Vurpillot et al., who focus on the numerical methods used to understand, treat, and potentially correct imaging issues commonly affecting the data in atom probe experiments. D. Haley et al. present a new tool for the simulation of 3D tomographic volumes, using advanced level set methods. In the article by C. Oberdorfer et al., the authors use a combined finite-element and molecular dynamics simulation approach that models the field evaporation process to show the effects of athermal relaxation on solute migration in the Al-Cu system. S. Katnagallu et al. use machine learning approaches and advanced image processing routines for data extraction in field ion microscopy. Finally, C. Ciu and S. Ringer provide a review of the opportunities and challenges in combining atom probe microscopy and density functional theory in materials science.

The use of computers continues to enable advanced understanding of both physical mechanisms that underpin the atom probe technique and deeper understanding of the nature of atom probe tomography data. We hope that you find these articles contained in this volume to be both stimulating and practically useful.

\section{References}

[1] M.K. Miller, R.G. Forbes, Atom-Probe Tomography: The Local Electrode Atom Probe, 1st ed., Springer US, Boston, MA, 2014. http://link.springer.com/10.1007/978-1-4899-7430-3 (accessed November 17, 2016).

[2] J.J. Hren, S. Ranganathan, Field-Ion Microscopy, Plenum Press, New York, 1968.

[3] A.J.W. Moore, The Simulation of FIM Desorption Patterns, Philosophical Magazine A. 43 (1981) 803-814.

[4] T.M. Hall, A. Wagner, D.N. Seidman, A computer-controlled time-of-flight atom-probe field-ion microscope for the study of defects in metals, J. Phys. E: Sci. Instrum. 10 (1977) 884.

[5] J.A. Panitz, R.J. Walko, Determination of nanosecond high-voltage pulse shapes at the surface of 
needle emitters, Rev. Scientific Instruments. 47 (1976) 1251-1254.

[6] H.F. Liu, T.T. Tsong, Review of Scientific Instruments. 55 (1984) 1779.

[7] P.P. Camus, D.J. Larson, T.F. Kelly, Simulation of Rapid Thermal Pulsing for Field Evaporation, Applied Surface Science. 67 (1993) 467-472.

[8] A. Cerezo, P.H. Clifton, G.D.W. Smith, Specimen Temperature Rises in a Nanosecond Laser Pulsed 3-D Atom Probe, Microscopy and Microanalysis. 11 (2005) 878-879.

[9] B. Gault, A. Vella, F. Vurpillot, A. Menand, D. Blavette, B. Deconihout, Optical and Thermal Processes Involved in Ultrafast Laser Pulse Interaction with a Field Emitter, Ultramicroscopy. 107 (2007) 713-719.

[10] F. Vurpillot, J. Houard, A. Vella, B. Deconihout, Thermal response of a field emitter subjected to ultra-fast laser illumination, J. Phys. D: Appl. Phys. 42 (2009) 125502.

[11] M. Tsukada, H. Tamura, K.P. McKenna, A.L. Shluger, Y.M. Chen, T. Ohkubo, K. Hono, Mechanism of laser assisted field evaporation from insulating oxides, Ultramicroscopy. 111 (2011) $567-570$.

[12] M. Karahka, H.J. Kreuzer, Field evaporation of oxides: A theoretical study, Ultramicroscopy. 132 (2013) 54-59. doi:10.1016/j.ultramic.2012.10.007.

[13] L.T. Stephenson, M.P. Moody, P.V. Liddicoat, S.P. Ringer, New Techniques for the Analysis of Fine-Scaled Clustering Phenomena within Atom Probe Tomography (APT) Data, Microscopy and Microanalysis. 13 (2007) 448-463.

[14] B.P. Geiser, T.F. Kelly, D.J. Larson, J. Schneir, J.P. Roberts, Spatial Distribution Maps for Atom Probe Tomography, Microscopy and Microanalysis. 13 (2007) 437-447.

[15] E.A. Marquis, J.M. Hyde, Applications of atom-probe tomography to the characterisation of solute behaviour, Materials Science and Engineering R. 69 (2010) 37-62.

[16] F. Vurpillot, C. Oberdorfer, Modeling Atom Probe Tomography: A review, Ultramicroscopy. 159, Part 2 (2015) 202-216. doi:10.1016/j.ultramic.2014.12.013. 